\title{
General approach to optimize settings of ship radars repair procedures
}

\author{
Evgeni Guglev",*, and Chavdar Alexandrov ${ }^{2}$ \\ ${ }^{1}$ Nikola Vaptsarov Naval Academy, Faculty of Navigation, 9026 Varna, Bulgaria \\ ${ }^{2}$ Nikola Vaptsarov Naval Academy, Department of Electronics, 9026 Varna, Bulgaria
}

\begin{abstract}
A number of possible formal methods for constructing an approach to solve the problem of global optimization of the setting process of ship radars repair procedure are introduced in this paper. The basis of the formalized task assignment is the selection of a sequence of operations related to determining the corresponding values of the electronic (electrical) components of radars, providing the global optimum of the created imitation model of the whole task with the corresponding subtasks. The algorithm of the global optimization task is built in the form of imitation experimental procedures with a multi-alternative optimization model. The multi-plan character (taking into account the different ranges) and the multi-criteria of such an experiment require an automatic search for optimizing the scanning at different ranges. The alternative for inclusion in the relevant group, with good result achieved is set in accordance with expert estimations (for the time of the scanning), Boolean variables (alternative for the inclusion of different ranges) or stochastic variables - by nomograms. The six-steps method (for six different ranges) is presented as a sequence of achieved optimums for the different ranges. The optimal set of functions determined by the task solution method is a solution of a stochastic problem including scanning time, pulse duration and ranges.
\end{abstract}

\section{Introduction}

The formalized decision-making task assignment (determining the optimal values of $R^{*}, C^{*} \ldots$ ) for different ranges is considered as a multi-alternative, multi-criterion optimization model of a complex system [1, 2, 3, 4]. For the individual levels (ranges), whose sequence is determined under T-algorithmically constrained limits, the exact $L$ (scanning) value for "long" ranges depends on the specified $L$ optimum for "small" ranges. The approach is a combination of a priori (expert assessments and different nomograms), axiomatic (person adopting a solution) and adaptive (stochastic) approximation. It allows for alignment within the entire optimization procedure, movement both in algorithmically defined and dependent on one another levels $X_{i}$ (for different ranges) and optimization of scanning time of the appropriate ranges $L_{j}=f\left[T\left(R^{*}\right), T\left(C^{*}\right)\right]$ at repair,

where: $R^{*}$ (Setup Resistance) and $C^{*}$ (Setup Capacity) are to be considered as stochastic quantities.

\section{Formulation of the problem}

The process of task assignment is done as described below.

Dependence $L=f(T)$ for the different ranges and dependence $T=f\left(R^{*}\right), T=f\left(C^{*}\right)$ given in [5] are experimentally predefined. Here $T$ is pulse duration.
Fig. 1 indicates the respective specified resistant tolerances for the different ranges.

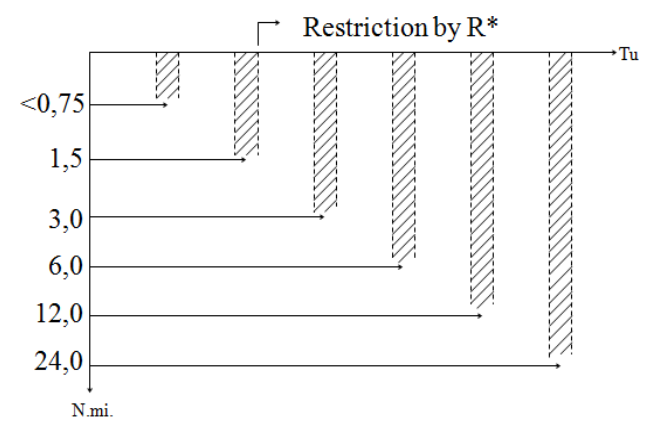

Fig. 1. Resistance tolerance for different ranges.

1. By multi-alternative optimization in Fig. 2 is set the optimization sequence for the different ranges shown in Fig. 1.

2. The accurate limits of the scanning are precised by the person making decision (PMD). This person determines the corresponding actual duration of the scanning required for the procedure in Fig. 3 (structural diagram of multi-criteria optimization).

3. In Fig. 3, using the method described in [5], the $L_{o p t}$ and the corresponding $T_{\text {opt }}$ are defined by the nomogram $L=f(T)$.

4. In Fig. 3, applying the same method by nomogram $T=f\left(R^{*}\right), T=f\left(C^{*}\right)$ follows determination of the optimal value of $R$ and $C$.

\footnotetext{
$\overline{{ }^{*} \text { Corresponding author: egigugi@abv.bg }}$
} 


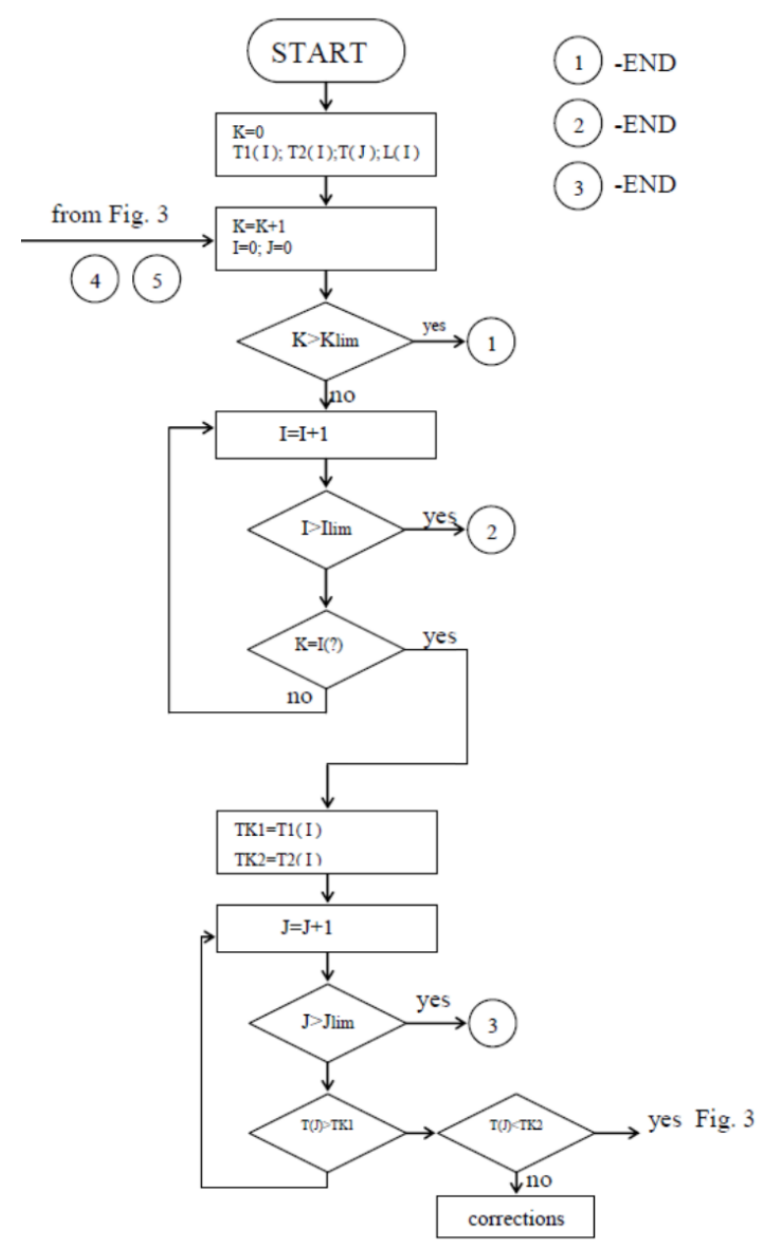

Fig. 2. Multi-alternative optimization.

\section{Essentiality of the implemented survey approach}

The multi-plan character of such an experiment require takes into account the different ranges and the multicriteria of such an experiment require an automatic search to optimize the scanning at different ranges providing the extreme value of the target function [6], i.e.:

$$
\Psi\left[X_{i}, L_{j}\left(T_{j}\right), T_{k}\left(R^{*}\right), T_{k}\left(C^{*}\right)\right] \rightarrow \min ,
$$

where $X_{i}$ is Boolean variable, implementing the appropriate range when the corresponding limitations are met.

$$
\text { 1) }
$$

$$
X_{i}=1
$$

at:

a) $i \equiv j-$ at a certain range there is a matching of exactly $j$ from a certain combination $L_{j}\left(T_{j}\right)$, for each radar scale from 1 to 6 (see Fig.1);

b)

$$
T_{1}(i)<T_{j}<T_{2}(i),
$$

where $T_{j}$ are limitation rows described in [5];

c) Performance of other conditions;

$$
X_{i}=0
$$

in case of non-fulfillment of any of the conditions $a, b, c$.

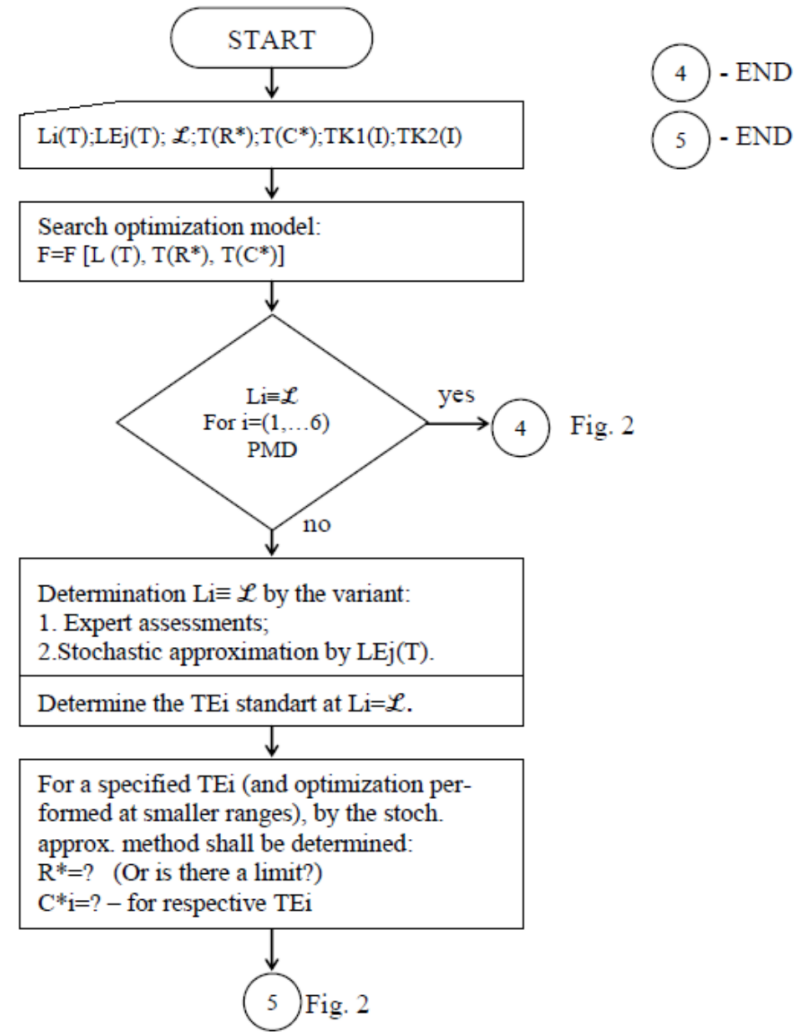

Fig. 3. Structural diagram of multi-criteria optimization.

$L\left[T\left(R^{*}\right), T\left(C^{*}\right)\right] ; L_{j}\left(T_{j}\right)$ - require multi-criteria optimization;

$L_{j} ; T_{j} ; R^{*} ; C^{*}-$ Stochastic quantity [5];

$L_{j} ; T_{j}$ - Current non-optimal values for each separate radar scale (range).

\section{Steps of optimization}

\section{Step 1: A priori research}

For a certain level, according to the realization of the stochastic quantity $L$, the $L_{i}(T)$ is determined either by $T=T\left(R^{*}\right)$ or by $T=T\left(C^{*}\right)$.

$R^{*}, C^{*}$ change using the rules: $T=\alpha R^{*} C^{*}$ - given in [5], and determining $L_{i}=L_{i}(T)$.

Performing multi-alternative optimization (for different levels or corresponding ranges) and multicriteria optimization (for each level separately) lead to a global optimum [7].

\section{Step 2: It is done by using decision-makers (PMD)}

The question set to the PMD is, "Are all the parameters of the $L_{i}$ scanning satisfactory?"

In case of a negative response, the possibility is analyzed:

1. $\quad \Psi_{i} L=f(\Delta L)=f[\Delta L(T)] \rightarrow \min$.

After reaching an optimum by $L, T_{\text {opt }}$ is determined and is accepted that $T_{\text {standatd }}=T_{\text {opt }}$.

2. $\Psi_{i} T=f^{\prime}(\Delta T)=f^{\prime}\left[\Delta T\left(R^{*}\right), \Delta T\left(C^{*}\right)\right] \rightarrow \min$. 
After reaching an optimum by $T, R_{o p t}, C_{o p t}$ are determined to achieve optimal scanning.

$\Delta L$ - deviation from the standard value of the scanning $\mathscr{L}$, $\Delta T$ - deviation from $T_{\text {opt }}$ defined in [5].

\section{Step 3: Going to an optimum}

A setting of the values is done:

1.

$$
L^{r+1}=L^{r}+\Delta L(T)
$$

$L^{*}=\mathscr{L}^{\text {is determined at }}$

$$
\Delta \Psi_{i L} \rightarrow \min ,
$$

where $L$ is scanning,

$\mathscr{L}$ - set reference,

$L^{*}$ - optimal scanning, which determines the corresponding $T_{\text {opt }}$ on the nomogram $L(T)$.

2. An exact (optimal) reference is defined $T_{E}=T_{\text {opt }}$

$$
T^{r+1}=T^{r}+\Delta T\left(R^{*}\right)+\Delta T\left(C^{*}\right)
$$

$T^{*}=T_{E}$ is determined at

$$
\Delta \Psi_{i T} \rightarrow \min .
$$

Next follows the determination of $R^{*}, C^{*}$.

$$
R^{r+1}=R^{r}+\Delta R
$$

The optimal $R^{*}$ is defined using the method of [5].

$$
C^{r+1}=C^{r}+\Delta C
$$

The optimal $C^{*}$ is defined using the same method.

\section{First procedure - Task 1}

It is demonstrated in Fig. 2.

If the pulse duration does not fall within any of the restriction intervals for the different ranges, then a correction is made by the PMD.

\section{Second procedure - Task 2}

After a successful test of the first procedure in Fig. 2 a second procedure is performed - the multi-criteria optimization of Fig. 3. This procedure leads to determination of the optimum, which is the above mentioned Step 3.

It is possible to increase the speed of convergence at the expense of the management of the choice by means of objective (by degree of change of the target function) and subjective (according to PMD information) localprognostic estimations of the direction of movement to extremum.

The described dialogue procedure uses the experience of the expert, his ability to work with abstract and fuzzy concepts in conducting automated analysis with multiple alternative optimization models [8].

\section{Experiments}

The experiments are organized under the following conditions:

1. The nomograms $L_{i}\left(T_{i}\right)(i=1 \div 6$ for each radar scale $)$ and $T\left(R^{*}\right), T\left(C^{*}\right)$ are determined in advance.

2. The current measured $L_{i}, T_{i}(i=1 \div 6$ for each radar scale) have to be tuned. (see Fig.1).

3. Using the expert assessments or PMD, $L_{i} \& \mathscr{L}$ are compared and the path of decision is determined.

4. Solution of Task 1: "Multi-alternative optimization at algorithmically constrained limitations" - according to Fig. 2.

Fig. 1 gives the respective permissible intervals.

Rows $T_{1}(i), T_{2}(i)$ are pre-defined under the Technical Conditions (the corresponding pairs give the margin for the respective levels).

5. Solution of Task 2 ("Multi-criteria optimization" according to Fig. 3.):

$$
\Delta \Psi_{i L} \rightarrow \min , \text { at } L_{i} \equiv \mathscr{L} \text { we get } T_{i e}=T_{o p t},
$$

where $T_{i e}$ is the reference;

$$
\Delta \Psi_{i T} \rightarrow \min , \text { at } R^{*} \equiv R_{o p t}
$$

or $C^{*} \equiv C_{\text {opt }}$ or both at $T_{i}=T_{i e}$.

6. After each successful test in Fig. 3, the specified and the actual $L_{i}\left(T_{i}\right)$ are compared by PMD and the decision is taken.

7. Additional optimization (if required):

- The dependence $L=f(T)$ is determined experimentally.

- There is an a priori data on the dependences $T=f\left(R^{*}\right)$, $T=f\left(C^{*}\right)$ given in [5].

\section{Conclusions}

The algorithms described in this research make it possible to completely exclude the "active" participation of a person in complete setup of the radar repairing process.

The research provides a general solution to the task of obtaining optimal values of the radar parameters.

By using information obtained from the optimization of the radar setup process, real applicability is ensured.

The developed algorithms, i.e. "instrumentation" can be used to repair ship radars.

This study has been carried out under project BG05M2OP0012.009-0037-C01 "Support for the development of PhD students, post-doctoral students, young scientists and lecturers at the Nikola Vaptsarov Naval Academy", with Nikola Vaptsarov Naval Academy, Varna as beneficiary. The project is funded by the Intelligent Growth Science and Education Operational Program, co-financed by the European Union through the European structural and investment funds. 


\section{References}

1. A. Donets, Y. Lvovich, V. Florov, Automatic analysis and optimization of radio equipment constructions and technologies, in Russian, Moscow (1983)

2. C. Floudas, P. Pardalos, Encyclopedia of optimization, Dordlecht (Kluwer Acad. Pub., 2001)

3. Z. Nikolov, M. Tsvetkov, T. Tsanev, Experimental research of a jamming over a direct sequence spread spectrum system, Journal of Marine Technology and Environment, 2, 83-88 (2009)

4. Y. Sivkov, Building maritime data hub by using Arduino IoT platform, IAMU AGA 2017, Varna, I, 533-540 (2017)

5. E. Guglev, Y. Dachev, Stochastic approach to optimize the setting of radiolocation stations at repair, Journal of Marine Technology and Environment, Constanta, 2, 33 - 37 (2017)

6. N. Madjarov, L. Michailova, Adaptive multiple model algorithms for monitoring and control of stochastic systems, Journal of Automatics and Informatics, in Bulgarian, 2 (1998)

7. S. Stoyanov, Methods and algorithms for optimization, in Bulgarian, Sofia (Technica, 1990)

8. P. Pardalos, R. Horst, Introduction to global optimization, Norwood (Kluwer Acad. Pub., 1995) 